\title{
Perioperative pulmonary rehabilitation training (PPRT) can reduce the cost of medical resources in patients undergoing thoracoscopic lung cancer resection: a retrospective study
}

\author{
Min Kong ${ }^{1,2}$, Hongkui Zheng ${ }^{1}$, Lulu Ding ${ }^{3}$, Ke Jin ${ }^{1,2}$, Jianfei Shen $^{1}$, Minhua Ye ${ }^{1,2}$, Baofu Chen ${ }^{1}$ \\ ${ }^{1}$ Department of Thoracic Surgery, Taizhou Hospital of Zhejiang Province affiliated to Wenzhou Medical University, Taizhou, China; ${ }^{2}$ Department of \\ Thoracic Surgery, Enze Hospital, Taizhou Enze Medical Center (Group), Taizhou, China; ${ }^{3}$ Central Sterile Supply Department, Taizhou Hospital of \\ Zhejiang Province affiliated to Wenzhou Medical University, Taizhou, China \\ Contributions: (I) Conception and design: M Kong, M Ye; (II) Administrative support: B Chen; (III) Provision of study materials or patients: M Ye, \\ K Jin; (IV) Collection and assembly of data: H Zheng, L Ding; (V) Data analysis and interpretation: M Kong, J Shen; (VI) Manuscript writing: All \\ authors; (VII) Final approval of manuscript: All authors. \\ Correspondence to: Minhua Ye. Department of Thoracic Surgery, Taizhou Hospital of Zhejiang Province affiliated to Wenzhou Medical University, \\ Taizhou, China. Email: ymh@enzemed.com; Baofu Chen. Department of Thoracic Surgery, Taizhou Hospital of Zhejiang Province affiliated to \\ Wenzhou Medical University, Taizhou, China. Email: chenbf@enzemed.com.
}

Background: To evaluate the benefits of perioperative pulmonary rehabilitation training (PPRT) in patients undergoing thoracoscopic lung cancer resection.

Methods: The clinical data of 1,427 patients undergoing thoracoscopic lung cancer resection were collated. Of these patients, 779 received PPRT (the PPRT group), which included systematic education, improvement of posture, diaphragmatic respiration, bilateral lower thoracic expansion, surgical side thoracic local expansion, incentive spirometry training, effective cough training, aerobic walking, and other systematic pulmonary rehabilitation training. The other 648 patients did not receive PPRT (the non-PPRT group). Baseline characteristics including length of hospital stay, cost of hospitalization, and the incidence of postoperative pulmonary complications (PPCs) were assessed.

Results: There was no significant difference between the PPRT group and the non- PPRT group in terms of age, gender distribution, tumor location, operation mode, postoperative pathological type, TNM stage, and other baseline characteristics $(\mathrm{P}>0.05)$. The complication index of the PPRT group was slightly higher than that of the non-PPRT group $(\mathrm{P}<0.05)$. Patients in the PPRT group had significantly fewer postoperative hospitalization days (PHD) $\{6.1$ days [95\% confidence interval (CI): 5.8 to 6.4] vs. 6.4 days (95\% CI: 6.1 to 6.7), P=0.002 \}, fewer total hospitalization days (THD) [9.3 days (95\% CI: 8.9 to 9.7) vs. 10.8 days (95\% CI: 10.3 to 11.3$), \mathrm{P}=0.000]$, lower non-surgical expenses $(35,024 \pm 9,742$ vs. $36,831 \pm 10,245$ $\mathrm{RMB})$, and fewer cases of PPCs) (3.72\% vs. 6.33\%, $\mathrm{P}=0.016)$ compared to patients in the non-PPRT group. In the subgroup analysis, patients less than 60 years old in the PPRT group fared better in terms of the PHDs, total inpatient days, and non-surgical expenses compared to patients in the non-PPRT group $(\mathrm{P}<0.05)$. In patients aged 60 years and older, the THDs in the PPRT group was less than that in the non-PPRT group $(\mathrm{P}<0.05)$, but there were no significant differences in the PHDs and non-surgical expenses.

Conclusions: PPRT can reduce the cost of medical resources in patients undergoing thoracoscopic lung cancer resection, especially by shortening the length of hospital stay, reducing the cost of hospitalization, and reducing PPCs.

Keywords: Pulmonary rehabilitation; lung cancer; thoracoscope; hospital costs

Submitted Jan 21, 2021. Accepted for publication Apr 01, 2021.

doi: $10.21037 /$ apm-21-478

View this article at: http://dx.doi.org/10.21037/apm-21-478 


\section{Introduction}

Lung cancer has one of the highest incidences and mortality rates of all malignancies in the world (1). Surgical anatomic resection of lung lesions plus systematic lymph node dissections are important methods for the treatment of lung cancer. In the past 30 years, advances in video-assisted thoracoscopic surgery (VATS) for lung cancer treatment has resulted in surgery that is minimally invasive, with reduced postoperative pain and other discomfort. This, in turn, has helped to improve postoperative recovery for the patient and reduced the cost of medical resources $(2,3)$.

However, many patients have an underlying lung condition. Approximately two-thirds of men and half of women present with chronic obstructive pulmonary disease (COPD) at the time of lung cancer diagnosis (4). Consequently, some patients have poor cardiopulmonary endurance and cannot tolerate surgical anesthesia, resulting in prolonged waiting times for VATS. Conversely, patients who undergo VATS can still experience pain, increased airway secretions, and decreased respiratory efficiency (5-7). Patients with severe diseases may suffer from atelectasis, pulmonary infection, and other postoperative pulmonary complications (PPCs). In fact, the incidence of PPCs ranges from $2 \%$ to $40 \%(8,9)$, and the risk of PPCs increases significantly in elderly patients, and patients with a history of smoking, low cardiopulmonary function, and pulmonary disease. PPCs is one of the main contributors to reduced quality of life. It also prolongs the length of hospital stays and even affects postoperative survival after pneumonectomy. Accordingly, it is closely related to the mortality rate and medical expenses (10-13).

Therefore, improving preoperative lung function and preventing and controlling the occurrence of PPCs plays an important role in the surgical outcomes and postoperative rehabilitation of patients with VATS lung cancer resection. Perioperative pulmonary rehabilitation training (PPRT) is a comprehensive intervention measure to give individualized treatment under the condition of fully evaluating patients. Such treatment, which mainly includes exercise intervention, behavior intervention, and health education. Its purpose is to improve the physiological and psychological state of patients with chronic lung disease through a variety of personalized interventions, so as to promote the maintenance of healthy behaviors. As a non-drug-assisted treatment method, PPRT has been increasingly applied in clinical practice because it can improve lung function in patients to a certain extent, effectively reducing postoperative complications (14). There was a similar report includes a total of 208 chronic obstructive pulmonary disease (COPD) patients participated in a PPRT program, and their report shows that improvements in exercise capacity and quality of life were seen following PPRT both before and after thoracic surgery (15).

PPRT speeds up hospitalization turnover, and thus reduces hospitalization costs. However, there is still a lack of large-sample clinical data to support its application. This study retrospectively analyzed the data of 1,427 patients in a single center to evaluate the benefits of PPRT in improving patient outcomes and finally reducing the cost of medical resources in patients undergoing thoracoscopic lung cancer resection.

We present the following article in accordance with the STROBE reporting checklist (available at http://dx.doi. org/10.21037/apm-21-478).

\section{Methods}

\section{Data source and study population}

The clinical data of patients undergoing lung cancer resection in the Department of Thoracic Surgery of Taizhou Hospital, Zhejiang Province, between June 2018 and February 2020 were collated. The center commenced routine perioperative lung rehabilitation training for patients undergoing thoracic surgery from April 2019 to February 2020 for a total of 10 months. Therefore, the clinical data of patients undergoing lung cancer resection in the center from June 2018 to March 2019 were used as the control group.

The inclusion criteria were as follows: (I) pathological diagnosis of lung cancer; (II) thoracoscopic surgery (including conversion to open surgery); and (III) complete clinical information available. The exclusion criteria were as follows: (I) open surgery; (II) pneumonectomy; (III) reoperation; (IV) serious postoperative complications; and (V) admission to the intensive care unit. According to the inclusion and exclusion criteria, a total of 1,427 cases were included in this retrospective study, with 779 patients who underwent perioperative pulmonary rehabilitation training (the PPRT group), and 648 patients who did not receive perioperative pulmonary rehabilitation training (the nonPPRT group).

All procedures performed in this study involving human participants were in accordance with the Declaration of 
Helsinki (as revised in 2013). This study was approved by the ethics committee of Enze Hospital of the Taizhou Enze Medical Center (group) in Zhejiang province, China. As this was a retrospective study, informed consent was not required.

\section{PPRT program}

The preoperative and postoperative training programs are listed below. The details for each specific operation are provided in Appendix 1.

PPRT involved the following:

(I) The systematic education and demonstration was provided by the cardiopulmonary rehabilitation physician division. The perioperative lung rehabilitation manual was issued and explained to ensure the patients were familiar with it, and a demonstration training session lasting more than 30 minutes was provided at least once.

(II) Respiratory training included diaphragmatic breathing, thoracic dilated breathing, local dilated breathing of the lungs, and respiratory training device training. Each exercise was performed in sets of 15 repeats, 3 to 6 times a day. The sets could be performed consecutively, or with a rest period in between.

(III) Patients were expected to practice an effective way of coughing and expectoration repeatedly and be familiar with the process.

(IV) Exercise therapy included ankle pump exercises and limb flexibility training (chest expansion, shoulder joint activities, and axis bending exercises). Each action was performed in sets of 10 repeats, with 5 sets performed every day. Aerobic exercise included walking (400-500 meters in 10 minutes as the target speed and $20-30$ minutes as the termination target) 3 to 6 times a day, and stair climbing training (between 15-30 minutes) once a day.

Postoperative pulmonary rehabilitation training involved the following:

(I) Respiratory training included diaphragmatic breathing, thoracic dilated breathing, local dilated breathing of the lungs, and respiratory device training. Each exercise was performed in sets of 15 repeats, 3 to 6 times a day. The sets could be performed consecutively, or with a rest period in between.
(II) Effective cough and expectoration, 8-10 times per set, 1 set every 2 hours except while sleeping.

(III) Exercise therapy including body position management (mainly in a recumbent position), ankle pump exercises, and limb flexibility training (chest expansion, shoulder joint activities, and axis bending exercises). These exercises were performed in sets of 10 repeats, with 5 sets per day. Patients were also required to walk, beginning with 5 10 minutes of out-of-bed activity, 2 to 3 times a day, and gradually transitioning to walking for 10 minutes, and then extending that time to 20 30 minutes.

\section{Observation indicators}

The baseline characteristics examined included age, gender distribution, comorbidity, tumor location, operation mode, pathological diagnosis, and tumor stage. The comorbidities were assessed by the Charlson comorbidities index (CCI). The higher the score, the more complications $(16,17)$. The surgical methods included VATS lobectomy, local resection, and lobectomy plus local resection. Each case of primary lung cancer also underwent systematic lymphadenectomy at the same time.

The length of stay in hospital, non-surgical expenses, and PPCs were also analyzed. The length of stay in hospital included total hospitalization days (THD) and postoperative hospitalization days (PHD). Non-surgical expenses during hospitalization are defined as total hospitalization expenses minus surgical expenses. Details of PPCs were collated using the electronic medical records system. Pulmonary infection is a PPC and is defined according to the following criteria: (I) chest computed tomography (CT) or chest film showing pulmonary exudation and consolidation; (II) fever above $38^{\circ} \mathrm{C}$; (III) white blood cell (WBC) count $>10,000$ or $<3,000 / \mathrm{mm}^{3}$; and (IV) pathogenic bacteria or purulent secretion in the sputum or bronchoscopy. Other PPCs included atelectasis of the lung lobe or whole lung as confirmed by chest CT or chest film; postoperative pneumothorax as indicated by chest CT or chest film greater than $30 \%$; and postoperative pleural effusion defined as pleural effusion greater than that indicated by the chest $\mathrm{CT}$ or chest $\mathrm{X}$-ray.

\section{Statistical analysis}

Statistical analysis was performed using the SPSS 25.0 
software. For continuous variables, descriptive statistics were used to calculate the number of observations (n), mean/median, minimum and maximum, and the $95 \%$ confidence interval (CI) of variables. A $t$-test or nonparameter test (Wilcoxon) was used for comparison between the two groups. For discontinuous variables (classified variables), the number and frequency (percentage) of occurrence of each category was calculated by descriptive statistics, and the comparison between the two groups was conducted by Pearson chi square test or Fisher's exact test. $\mathrm{P}<0.05$ was considered statistically significant.

\section{Results}

\section{A comparison of baseline patient characteristics}

There was no significant difference between the two groups in terms of age, gender distribution, tumor location, operation mode, pathological diagnosis, tumor stage, and other baseline characteristics $(\mathrm{P}>0.05)$. The rehabilitation group had more complications and a higher CCI index score compared to the non-rehabilitation group $(\mathrm{P}<0.05)$ (Table 1).

\section{A comparison of hospitalization cost, time, and postoperative complications}

THD, PHD, non-surgical expenses, and PPC in the rehabilitation group were significantly lower compared to that observed in the non-rehabilitation group $(\mathrm{P}<0.05$; Table 2; Figure 1A,B).

\section{Subgroup analysis}

Taking 60 years of age as the boundary, the sub-group stratified analysis was carried out for the population in the two groups. First, there was no statistical difference in the age composition between the two groups $(\mathrm{P}>0.05$; Table 3). Furthermore, in the group of patients younger than 60 years old, those in the rehabilitation group fared significantly better than those in the non-rehabilitation group in terms of PHD, THD, and non-surgical expenses $(\mathrm{P}<0.05$, Table 4; Figure $1 C, D$ ). In the group of patients older than 60 years, the rehabilitation group had significantly fewer THD compared to patients in the non-rehabilitation group $(\mathrm{P}<0.05$; Table 5; Figure 1C). The rehabilitation group also had slightly fewer PHD and lower non-surgical expenses compared to the non-rehabilitation group, but there was no statistical difference $(\mathrm{P}>0.05$; Table 5, Figure 1D).

In the rehabilitation training group, the majority of patients were given guided rehabilitation training either once or twice $(775 / 779,99.5 \%)$. However, there were no statistical differences in the PHD, THD, PPC, nor the nonsurgical expenses between patient who received 1 session of guided training and those who received 2 sessions of guided training $(\mathrm{P}>0.05$; Table 6).

\section{Discussion}

Common reasons for the prolongation of THDs and the increased cost of lung cancer resection include the postponement of the scheduled operation due to poor pulmonary function prior to the operation and the prolongation of hospitalization time caused by pulmonary complications after the operation. Thoracoscopic lung cancer resection, also known as VATS lung cancer resection, has been developed over the last 30 years. Although it is less invasive than traditional open surgery, postoperative pulmonary complications still exist. Some studies have shown that perioperative pulmonary rehabilitation may improve lung function and reduce the incidence of pulmonary complications in patients who have lung cancer resection (18-21). However, more randomized controlled studies with large sample sizes are still required so far to confirm the benefits of perioperative pulmonary rehabilitation. The objective and quantitative indicators in this current retrospective study revealed that perioperative lung rehabilitation training can be beneficial in terms of the rapid rehabilitation of patients with thoracoscopic lung cancer resection, thus reducing the cost of medical resources.

Pulmonary rehabilitation is a comprehensive intervention measure that provides individualized treatment by fully evaluating the patients. Such treatment includes exercise intervention, behavior intervention, and health education. Its purpose is to improve the physiological and psychological state of patients with chronic lung disease through a variety of personalized interventions, thereby promoting the maintenance of healthy behaviors (22). Pulmonary rehabilitation can improve the symptoms, quality of life, pulmonary function, and medical care utilization of patients with chronic respiratory diseases. Some reports have shown that perioperative pulmonary rehabilitation training, including preoperative and postoperative pulmonary rehabilitation training, can further improve a patient's operation tolerance and pulmonary function, and 
Table 1 A comparison of the baseline patient characteristics between the PPRT group and the non-PPRT group

\begin{tabular}{|c|c|c|c|}
\hline Characteristic & PPRT group $(\mathrm{N}=779)$ & Non-PPRT group (N=648) & $P$ value \\
\hline Gender (M/F) & $334 / 445$ & $293 / 355$ & 0.375 \\
\hline $\mathrm{CCl}$ & & & 0.018 \\
\hline 0 & $220(28.2 \%)$ & $203(31.3 \%)$ & \\
\hline 2 & $193(24.8 \%)$ & $151(23.3 \%)$ & \\
\hline 3 & 97 (12.5\%) & $56(8.6 \%)$ & \\
\hline$\geq 4$ & $43(5.5 \%)$ & $22(3.4 \%)$ & \\
\hline Tumor location & & & 0.413 \\
\hline Right lower lobe & $132(16.9 \%)$ & $134(20.7 \%)$ & \\
\hline Left upper lobe & $203(26.1 \%)$ & $149(23.0 \%)$ & \\
\hline Left lower lobe & $104(13.4 \%)$ & $83(12.8 \%)$ & \\
\hline Multiple lobes & $38(4.9 \%)$ & $26(4.0 \%)$ & \\
\hline Surgical methods & & & 0.134 \\
\hline VATS lobectomy & $387(49.7 \%)$ & $336(51.9 \%)$ & \\
\hline VATS lung segmental resection & $327(42.1 \%)$ & $258(39.8 \%)$ & \\
\hline VATS lobectomy + lung segmental resection & $36(4.6 \%)$ & $19(2.9 \%)$ & \\
\hline Lung metastases & $21(2.7 \%)$ & $21(3.3 \%)$ & \\
\hline Other & $30(3.9 \%)$ & $27(4.2 \%)$ & \\
\hline TNM classification & & & 0.404 \\
\hline Phase I & $354(45.4 \%)$ & $307(47.3 \%)$ & \\
\hline Phase II & $207(26.6 \%)$ & $153(23.7 \%)$ & \\
\hline Phase III & $187(24.0 \%)$ & $152(23.4 \%)$ & \\
\hline Phase IV & $10(1.3 \%)$ & $15(2.3 \%)$ & \\
\hline Lung metastases & $21(2.7 \%)$ & $21(3.3 \%)$ & \\
\hline
\end{tabular}

Except for age, all other variables are expressed as the number of cases (\%). CCI, Charlson comorbidities index; PPRT group, rehabilitation group; non-PPRT group, non-rehabilitation group; VATS, video-assisted thoracoscopic surgery; M, male; F, female. 
Table 2 A comparison of hospitalization days, cost, and postoperative complications between the PPRT group and the non-PPRT group

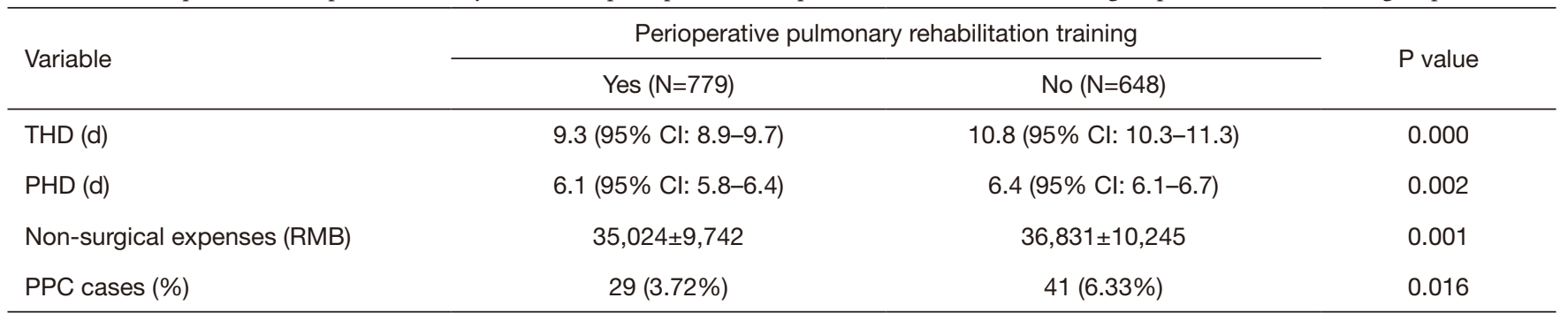

THD, total hospitalization days; PHD, postoperative hospitalization days; PPC, postoperative pulmonary complications; RMB, renminbi; $\mathrm{Cl}$, confidence interval.
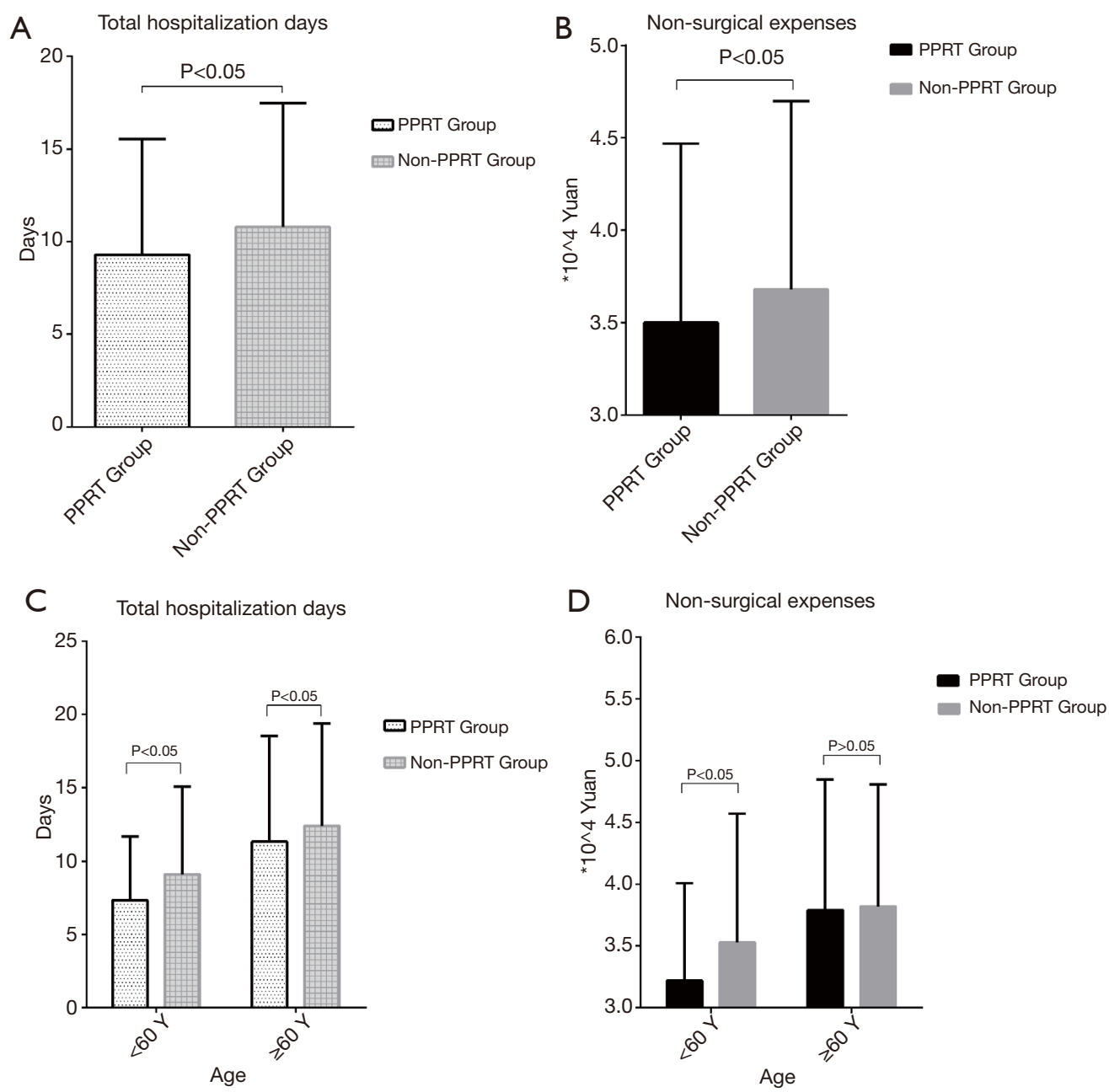

Figure 1 A comparison of total hospitalization days (THD) and non-surgical expenses (RMB) between the rehabilitation group (PPRT group) and the non-rehabilitation group (non-PPRT group). (A) Patients in the PPRT group had significantly fewer THD compared to patients in the non-PPRT group $(\mathrm{P}<0.05)$. (B) Patients in the PPRT group had significantly lower non-surgical expenses compared with patients in the non-PPRT group $(\mathrm{P}<0.05)$. (C) A comparison of the THD by age stratification showed that patients in the PPRT group had significantly fewer THD compared to the non-PPRT group irrespective of whether they were younger or older than 60 years of age $(\mathrm{P}<0.05)$. (D) A comparison of non-surgical expenses by age stratification showed a statistically significant difference between the PPRT group and the non-PPRT group when the patients were $<60$ years old $(\mathrm{P}<0.05)$, but no significant difference was found in patients aged $\geq 60$ years old $(\mathrm{P}>0.05)$. 
Table 3 A comparison of the age composition between the PPRT group and the non-PPRT group (P>0.05)

\begin{tabular}{|c|c|c|c|c|}
\hline Age & PPRT group $(\mathrm{N}=779)$ & Non-PPRT group ( $\mathrm{N}=648)$ & $\chi^{2}$ & $P$ value \\
\hline$\geq 60 y$ & $383(50.0 \%)$ & $331(51.1 \%)$ & & \\
\hline
\end{tabular}

PPRT group, rehabilitation group; non-PPRT group, non rehabilitation group.

Table 4 A comparison of patients aged $<60$ years in the PPRT group and the non-PPRT group

\begin{tabular}{|c|c|c|c|}
\hline Variable & \multicolumn{2}{|c|}{ Perioperative pulmonary rehabilitation training } & $P$ value \\
\hline THD (d) & 7.3 (95\% Cl: 6.9-7.8) & 9.1 (95\% Cl: 8.4-9.7) & 0.000 \\
\hline PHD (d) & 5.2 (95\% Cl: 4.9-5.6) & 5.6 (95\% Cl: 5.2-6.0) & 0.010 \\
\hline Non-surgical expenses (RMB) & $32,205 \pm 7,875$ & $35,315 \pm 10,368$ & 0.000 \\
\hline
\end{tabular}

THD, total hospitalization days; PHD, postoperative hospitalization days; RMB, renminbi; $\mathrm{Cl}$, confidence interval.

Table 5 A comparison of patients aged $\geq 60$ years in the PPRT group and the non-PPRT group

\begin{tabular}{|c|c|c|c|}
\hline Variable & \multicolumn{2}{|c|}{ Perioperative pulmonary rehabilitation training } & $P$ value \\
\hline THD (d) & 11.3 (95\% Cl: 10.6-12.0) & 12.4 (95\% Cl: 11.6-13.2) & 0.003 \\
\hline PHD (d) & 7.1 (95\% Cl: 6.6-7.6) & 7.2 (95\% Cl: 6.7-7.7) & 0.150 \\
\hline Non-surgical expenses (RMB) & $37,939 \pm 10,603$ & $38,282 \pm 9,927$ & 0.656 \\
\hline
\end{tabular}

THD, total hospitalization days; PHD, postoperative hospitalization days; RMB, renminbi; Cl, confidence interval.

Table 6 A comparison of patients who received one session of guided pulmonary rehabilitation training versus patients who received two sessions

\begin{tabular}{|c|c|c|c|}
\hline Variable & \multicolumn{2}{|c|}{ Number of guided pulmonary rehabilitation sessions } & $P$ value \\
\hline THD (d) & 9.1 (95\% Cl: 8.5-9.6) & 9.4 (95\% Cl: 8.8-10.1) & 0.536 \\
\hline PHD (d) & 6.0 (95\% Cl: 5.6-6.3) & 6.3 (95\% Cl: 5.9-6.8) & 0.878 \\
\hline Non-surgical expenses (RMB) & $35,287 \pm 8,871$ & $34,813 \pm 10,508$ & 0.497 \\
\hline
\end{tabular}

THD, total hospitalization days; PHD, postoperative hospitalization days; PPC, postoperative pulmonary complications; RMB, renminbi;

$\mathrm{Cl}$, confidence interval.

shorten the patient's hospitalization time (19-21). Studies have also shown that perioperative respiratory training can reduce the incidence of postoperative complications and improve pulmonary function and cough efficacy $(21,23)$. However, some researchers believe preoperative pulmonary function training can only improve pulmonary function, but not significantly reduce the incidence of postoperative complications (24). As an operation that is becoming increasingly recommended, thoracoscopic lung cancer resection has a conspicuous presence in the realm of cancer treatment. Compared with traditional thoracotomy, the biggest advantage of thoracoscopic lung cancer resection is that the incision is small and the damage to the chest wall tissue is limited, which is conducive to rapid postoperative 
recovery. However, the operation in the chest, including pneumonectomy, is actually similar to traditional open surgery, so the factors affecting postoperative recovery are similar. At present, there is still a paucity of research examining the benefits of pulmonary rehabilitation training in the perioperative period after resection. In this retrospective study, quantifiable indicators (such as length of stay and cost of hospitalization) were examined to determine the benefits of lung rehabilitation for patients with VATS lung cancer resection.

Our center is one of the earliest units to carry out thoracoscopic resection of lung cancer in China, with relatively mature surgical technology and processes. The patient population, including disease types, treated in our center are relatively constant, and is thus optimal for population matching. Beginning in April 2019, our center routinely carried out perioperative lung rehabilitation training for 10 months. This study compared the clinical baseline characteristics of patients who underwent lung cancer resection under thoracoscopy with or without perioperative lung rehabilitation training, and compared the differences in hospitalization cost, time, and PPCs. Considering that the cost of surgery and related consumables may bring bias to the comparison, non-surgical costs was selected as the observation index. Compared with the non-rehabilitation group, the rehabilitation group showed different degrees of benefits in terms of length of stay, cost of hospitalization, and PPCs. Considering that elderly patients with more basic lung diseases may benefit more from lung rehabilitation training, a comparative analysis was carried out based on the age of 60 years. The results showed that patients less than 60 years old benefited from lung rehabilitation training, while those aged 60 years and older only showed a significant benefit in terms of total hospital days, with no significant benefit observed in postoperative hospital days and non-surgical cost. This may suggest that short-term perioperative pulmonary rehabilitation training has a limited positive impact on the elderly patient who may have relatively greater trauma and weaker body function. However, it may also suggest that there is room for improving pulmonary rehabilitation training. This study also found there that there was no statistical difference between patients who were given guidance training once compared to patients who were give guidance training twice, suggesting that even one session of systematic guidance and training had a positive effect.

In patients who require lung cancer resection by thoracoscopy, rehabilitation training may improve the patient's lung function state, enhance their cardiopulmonary endurance, improve their effective expectoration efficiency, prevent thrombosis, and improve their psychological state, thereby promoting their rehabilitation, shortening hospitalization time, and reducing medical costs.

This study has some limitations. First, many other factors can contribute to the consumption of medical resources during thoracoscopic lung cancer resection. Since this is a retrospective study, it is not possible to completely exclude the bias caused by other confounding factors. Second, this study did not compare of some important indicators before and after lung rehabilitation, such as a lung function test, cardiopulmonary exercise test, and blood gas analysis, and these warrant further investigation by prospective randomized controlled clinical studies. Finally, this is a single-center study, and the reliability of the conclusions need to be verified by further multi-center trials.

This report demonstrated that perioperative pulmonary rehabilitation training can reduce the cost of medical resources in patients undergoing thoracoscopic lung cancer resection, especially by shortening the length of hospital stay, reducing the cost of hospitalization, and reducing PPCs. For relatively young patients $(<60$ years old), perioperative pulmonary rehabilitation training is also valuable. Guidance training provided by medical staff can improving the efficacy of pulmonary rehabilitation training in the perioperative period.

\section{Acknowledgments}

Funding: None.

\section{Footnote}

Reporting Checklist: The authors have completed the STROBE reporting checklist. Available at http://dx.doi. org/10.21037/apm-21-478

Data Sharing Statement: Available at http://dx.doi. org/10.21037/apm-21-478

Conflicts of Interest: All authors have completed the ICMJE uniform disclosure form (available at http://dx.doi. org/10.21037/apm-21-478). The authors have no conflicts of interest to declare.

Ethical Statement: The authors are accountable for all aspects of the work in ensuring that questions related 
to the accuracy or integrity of any part of the work are appropriately investigated and resolved. All procedures performed in this study involving human participants were in accordance with the Declaration of Helsinki (as revised in 2013). This study was approved by the ethics committee of Enze Hospital of the Taizhou Enze Medical Center (group) in Zhejiang province, China. As this was a retrospective study, informed consent was not required.

Open Access Statement: This is an Open Access article distributed in accordance with the Creative Commons Attribution-NonCommercial-NoDerivs 4.0 International License (CC BY-NC-ND 4.0), which permits the noncommercial replication and distribution of the article with the strict proviso that no changes or edits are made and the original work is properly cited (including links to both the formal publication through the relevant DOI and the license). See: https://creativecommons.org/licenses/by-nc-nd/4.0/.

\section{References}

1. Siegel RL, Miller KD, Jemal A. Cancer statistics, 2020. CA Cancer J Clin 2020;70:7-30.

2. Bendixen M, Jørgensen OD, Kronborg C, et al. Postoperative pain and quality of life after lobectomy via video-assisted thoracoscopic surgery or anterolateral thoracotomy for early stage lung cancer: a randomised controlled trial. Lancet Oncol 2016;17:836-44.

3. Berfield KS, Farjah F, Mulligan MS. Video Assisted Thoracoscopic Lobectomy for Lung Cancer. Ann Thorac Surg 2019;107:603-9.

4. Loganathan RS, Stover DE, Shi W, et al. Prevalence of COPD in women compared to men around the time of diagnosis of primary lung cancer. Chest 2006;129:1305-12.

5. Khoshbin E, Al-Jilaihawi AN, Scott NB, et al. An audit of pain control pathways following video-assisted thoracoscopic surgery. Innovations (Phila) 2011;6:248-52.

6. Steinthorsdottir KJ, Wildgaard L, Hansen HJ, et al. Regional analgesia for video-assisted thoracic surgery: a systematic review. Eur J Cardiothorac Surg 2014;45:959-66.

7. Ciftci B, Ekinci M, Celik EC, et al. Efficacy of an Ultrasound-Guided Erector Spinae Plane Block for Postoperative Analgesia Management After Video-Assisted Thoracic Surgery: A Prospective Randomized Study. J Cardiothorac Vasc Anesth 2020;34:444-9.

8. Harada H, Yamashita Y, Misumi K, et al. Multidisciplinary team-based approach for comprehensive preoperative pulmonary rehabilitation including intensive nutritional support for lung cancer patients. PLoS One 2013;8:e59566.

9. Canet J, Gallart L, Gomar C, et al. Prediction of postoperative pulmonary complications in a populationbased surgical cohort. Anesthesiology 2010;113:1338-50.

10. Epidemiology, practice of ventilation and outcome for patients at increased risk of postoperative pulmonary complications: LAS VEGAS - an observational study in 29 countries. Eur J Anaesthesiol 2017;34:492-507.

11. Serpa Neto A, Hemmes SN, Barbas CS, et al. Incidence of mortality and morbidity related to postoperative lung injury in patients who have undergone abdominal or thoracic surgery: a systematic review and meta-analysis. Lancet Respir Med 2014;2:1007-15.

12. Fernandez-Bustamante A, Frendl G, Sprung J, et al. Postoperative Pulmonary Complications, Early Mortality, and Hospital Stay Following Noncardiothoracic Surgery: A Multicenter Study by the Perioperative Research Network Investigators. JAMA Surg 2017;152:157-66.

13. Fleisher LA, Linde-Zwirble WT. Incidence, outcome, and attributable resource use associated with pulmonary and cardiac complications after major small and large bowel procedures. Perioper Med (Lond) 2014;3:7.

14. Vagvolgyi A, Rozgonyi Z, Kerti M, et al. Effectiveness of pulmonary rehabilitation and correlations in between functional parameters, extent of thoracic surgery and severity of post-operative complications: randomized clinical trial. J Thorac Dis 2018;10:3519-31.

15. Vagvolgyi A, Rozgonyi Z, Kerti M, Vadasz P, Varga J. Effectiveness of perioperative pulmonary rehabilitation in thoracic surgery. J Thorac Dis 2017;9:1584-91.

16. Charlson ME, Pompei P, Ales KL, et al. A new method of classifying prognostic comorbidity in longitudinal studies: development and validation. J Chronic Dis 1987;40:373-83.

17. Roffman CE, Buchanan J, Allison GT. Charlson Comorbidities Index. J Physiother 2016;62:171.

18. Sanchez-Lorente D, Navarro-Ripoll R, Guzman R, et al. Prehabilitation in thoracic surgery. J Thorac Dis 2018;10:S2593-600.

19. Benzo R, Wigle D, Novotny P, et al. Preoperative pulmonary rehabilitation before lung cancer resection: results from two randomized studies. Lung Cancer 2011;74:441-5.

20. Mujovic N, Mujovic N, Subotic D, et al. Preoperative pulmonary rehabilitation in patients with non-small cell lung cancer and chronic obstructive pulmonary disease. 
Arch Med Sci 2014;10:68-75.

21. Boujibar F, Bonnevie T, Debeaumont D, et al. Impact of prehabilitation on morbidity and mortality after pulmonary lobectomy by minimally invasive surgery: a cohort study. J Thorac Dis 2018;10:2240-8.

22. Spruit MA, Singh SJ, Garvey C, et al. An official American Thoracic Society/European Respiratory Society statement: key concepts and advances in pulmonary rehabilitation.

Am J Respir Crit Care Med 2013;188:e13-64.

23. Gao K, Yu PM, Su JH, et al. Cardiopulmonary

Cite this article as: Kong M, Zheng H, Ding L, Jin K, Shen J, Ye $M$, Chen B. Perioperative pulmonary rehabilitation training (PPRT) can reduce the cost of medical resources in patients undergoing thoracoscopic lung cancer resection: a retrospective study. Ann Palliat Med 2021;10(4):4418-4427. doi: 10.21037/apm$21-478$ exercise testing screening and pre-operative pulmonary rehabilitation reduce postoperative complications and improve fast-track recovery after lung cancer surgery: A study for 342 cases. Thorac Cancer 2015;6:443-9.

24. Nagarajan K, Bennett A, Agostini P, et al. Is preoperative physiotherapy/pulmonary rehabilitation beneficial in lung resection patients? Interact Cardiovasc Thorac Surg 2011;13:300-2.

(English Language Editor: J. Teoh) 


\section{Appendix 1}

\section{Key techniques for perioperative pulmonary rehabilitation training (PPRT)}

Diaphragmatic breathing: According to the patient's tolerance, he or she should assume a standing position, sitting position, or half-lying position, and relax the muscles of the whole body. The patient or therapist should place their hands on the chest and upper abdomen (the upper edge of the hand should be under the xiphoid process of the sternum) and encourage the patient to expand this area when he/she inhales deeply through the nose. When inhaling deeply, the thorax and upper abdomen (diaphragm position) will rise at the same time. It is important that the patient does not shrug when inhaling. Once the patient has inhaled to the maximum lung volume, he/she should hold their breath for 2-3 seconds. When exhaling, the chest and upper abdomen should contract inwards and downwards. The ratio of suction and exhalation is 1:3, or at least 1:2. In our study, 45 breaths were performed each time, in 3 sets of 15 repeats, with a 1-2-minute rest in between each set.

(1) Bilateral lower thorax expansion: According to the patient's tolerance, assume a standing position, sitting position, or half-lying position, and relax the muscles of the whole body. The patient or therapist should place their hands on the front and outer side of the 6th-10th quarter rib area, and the patient should be encouraged to expand this area when inhaling deeply through nose. It is important that the patient does not shrug when inhaling. Once the patient has reached the maximum lung volume, the patient should hold his/her breath for 2-3 seconds. When exhaling, the chest and upper abdomen should contract inwards and downwards. The ratio of suction and exhalation is 1:3, or at least 1:2. In our study, 45 breaths were performed each time in 3 sets of 15 repeats, with a 1-2-minute rest in between each set.

(2) Lung local expansion breathing: According to the patient's tolerance, he/she should assume a standing, sitting, or half-lying position, and relax the muscles of the whole body. The patient or therapist should place their hands on the surface projection of the surgical lung tissue, and the patient should be encouraged to expand this area while inhaling deeply through the nose. It is important that the patient does not shrug when inhaling. Once the patient has reached the maximum lung volume, the patient should hold his/her breath for 2-3 seconds. When exhaling, the surface projection of the surgical lung tissue and the upper abdomen should contract inwards and downwards. The ratio of inhalation to exhalation is 1:3, or at least 1:2. In our study, 45 breaths were performed each time, in 3 sets of 15 repeats, with a 1-2-minute rest in between each set.

(3) Effective cough training: According to the patient's tolerance, he or she should assume a standing, sitting, or halflying position. However, if possible, the patient should assume a forward-sitting position when coughing. The patient should relax all muscles in the body. The patient or therapist should place their hands on the chest and upper abdomen (the upper edge of the hand should be under the xiphoid process of the sternum) and encourage the patient to expand this area when inhaling deeply. Once maximum lung capacity is reached, the patient should hold their breath for 2-3 seconds, then suddenly contract the abdominal muscles inward, open the glottis, inhale deeply, and cough. When coughing after the operation, pay attention to protecting the mouth of the drainage tube with the hands to prevent pain and subcutaneous emphysema caused by excessive pressure. In this study, 3 sets of 10 repeats were performed over a 3-5-minute period. The patients were instructed to lie down every 3 hours, especially after a long session.

(4) Respiratory training device training: To use the incentive spirometer, the patient should assume a standing, sitting, or half-lying position depending on their tolerance. Patients should relax the muscles of the whole body. The mouthpiece should be placed in the mouth and the patient should inhale through the mouthpiece in a manner similar to the diaphragm breathing technique. According to the buoy visual inspection, low flow rate, and extended inspiratory time, the patient should aim to reach the age and gender target volume. Each session was divided into 3 sets of 10 repeats over a 2-3-minute period.

(5) Stair climbing training: Some patients were accompanied by medical staff. Patients were asked to adjust their breathing rhythm during exercise, such as breathe by shrinking the lips, exhale when exerting, avoid closing up. If there is obvious dyspnea, the patient should take a short rest and continue to exercise as soon as they feel ready. This was performed once a day, for 15-30 minutes.

(6) Aerobic walking: Under the monitoring of the pulse oximeter, the patient walks at a subjective and slightly faster pace than usual. In the event of chest distress, shortness of breath, etc., patients should slow down, take a proper rest, or stop walking depending on the monitoring situation. Prior to the operation, the target is to walk 400-500 $\mathrm{m}$ for 10 minutes each time, and the termination target is to walk 20-30 minutes each time. After the operation, the sessions 
should start with 10 minutes of walking each time, and gradually transition to 20-30 minutes each time.

(7) Ankle pump movement: I. Ankle flexion and extension movement: The patient lies flat or sits on the bed, with the lower limbs extended, the thighs relaxed, and the toes slowly hooked inside so as to face themselves as much as possible. Patients are asked to hold this position for 5-10 seconds, and then the toes will be stretched straight and pressed down to the maximum. Again, patients hold this position for 5-10 seconds, and relax. Patients should practice this 5-8 times every day for about 10 minutes. This can be carried out when the patient is awake, lying in bed, or having an infusion. II. Rotation of the ankle joint: The patient lies on his back or sits on the bed, stretches his legs, relaxes his thighs, centers on the ankle joint, and circles his toes 360 degrees, trying to maintain the maximum range of motion. Patients should practice this 5-8 times every day for about 10 minutes. This can be carried out when the patient is awake, lying in bed, or having an infusion. 